\title{
Topology Controlled Energy Proficient Protocol for Wireless Sensor Networks
}

\author{
Vibha Nehra, Ajay K.Sharma \\ Sharda University, Greater Noida, UP, INDIA \\ Vibha.nehra@sharda.ac.in \\ Dr. B R Ambedkar NIT, Jalandhar, Punjab, INDIA \\ sharmaajay@nitj.ac.in
}

\section{ABSTRACT}

\begin{abstract}
Random deployment in Wireless sensor networks lead to spatial node redundancy in close knit sensor networks. In this paper, an improved energy proficient PEGASIS based protocol (PEGASIS-TC) has been proposed. PEGASIS-TC exploits this spatial node redundancy by finding optimal subset of nodes that guarantee connectivity and turns off the remaining nodes based on $\mathrm{C}_{\mathrm{TR}}$ (Critical transmission range) to conserve energy which can be used in later stages to extend the network lifetime. The simulation results obtained show that PEGASIS-TC gives an increase in network lifetime as compared to PEGASIS.
\end{abstract}

\section{Indexing terms/Keywords}

Energy proficiency, PEGASIS, Spatial Node redundancy, Topology control.

\section{Academic Discipline And Sub-Disciplines}

Wireless sensor Network; Spatial Node Redundancy; Topology Control

\section{SUBJECT CLASSIFICATION}

Energy proficiency

\section{TYPE (METHOD/APPROACH)}

Simulation based

\section{Council for Innovative Research}

Peer Review Research Publishing System

\author{
Journal: INTERNATIONAL JOURNAL OF COMPUTERS \& TECHNOLOGY \\ Vol 11, No.8 \\ editor@cirworld.com
}

www.cirworld.com, member.cirworld.com 


\section{INTRODUCTION}

Materialization of affordable, portable, wireless communication \& computation devices, along with advances in communication infrastructure has led to rapid growth of mobile wireless networks. This has resulted in exponential growth of cellular networks, while on other side has led to renewal of interest of scientific and industrial community towards a scenario where a group of battery operated, radio equipped units communicate unattended without fixed infrastructure [1].

A wireless sensor network consists of a large number of sensor nodes deployed over a geographical area for monitoring physical phenomena like temperature, humidity, vibrations, seismic events, and so on [2]. Examples of scenarios where WSN can be used are described [1] in Estrin et al. [1999], Heinzelman et al. [1999], Khan et al. [2000], Mainwaring et al. [2002], Pottie and Kaiser [2000], Sadler et al. [2004], Schwiebert et al. [2001], Srivastava et al. [2001], Steere et al. [2000], and Szewczyk et al. [2004]. Each sensor node is a tiny device that includes three basic components: a sensing subsystem for data acquisition from the physical surrounding environment, a processing subsystem for local data processing and storage, and a wireless communication subsystem for data transmission to a central collection point (sink node or base station) [3]. In addition, a power source supplies the energy needed by the device to perform the programmed task.

Lifetime of Wireless Sensor Network may be defined as the time from the inception to the time when network becomes non-functional. A Network may become non-functional when a single node dies or when a particular percentage of nodes perish depending on application [4]. It is universally acknowledged that balanced energy dissipation for equalizing residual energy of nodes is one of the keys for extending network lifetime. Therefore, designing energy efficient protocols is important in the domain of data routing $[4,5]$

Experimental analysis has shown that data transmission is more expensive in terms of energy consumption, while data computation consumes significantly less [6]. The energy dissipation in transmitting a single bit of information is approximately the same as that required for processing thousand operations in a typical sensor node [7]. The energy dissipation in a sensing subsystem depends on the specific sensor type. Generally it is negligible with respect to the energy consumed by the processing and, above all, the communication subsystems. While in some cases, the energy consumption for data sensing may be comparable to, or even greater than, the energy needed for data transmission. In general, energy-saving techniques focus on two subsystems: the networking subsystem (i.e., energy management in the operations of each single node, as well as in the design of networking protocols), and the sensing subsystem (i.e., techniques are used to reduce the amount or frequency of energy-expensive samples) [2].

Here we focus on energy conservation in wireless sensor networks, specifically focusing on networking subsystem by considering duty cycling. The most effective energy-conserving operation is putting the radio transceiver in the (low-power) sleep mode whenever communication is not required. Ideally, the radio should be switched off as soon as there is no more data to send/receive, and should be resumed as soon as a new data packet becomes ready. In this way nodes alternate between active and sleep periods depending on network activity. This behavior is usually referred to as duty cycling, and duty cycle is defined as the fraction of time nodes are active during their lifetime [2]. Duty cycling can be achieved by exploiting node redundancy, which is typical in sensor networks. Adaptively selecting a minimum sub-set of nodes to remain active to ensure connectivity. Nodes that are not currently required for connectivity can go to sleep and save energy. Finding optimal subset of nodes that guarantee connectivity is referred to as topology control [1]. So, the basic concept behind topology control is to exploit the network redundancy to prolong the network longevity, typically increasing the network lifetime by a factor of 2-3 with respect to a network with all nodes always on [8, 9, 10].

The work in this paper introduces a PEGASIS [11] based protocol implementing the concept of Topology control called PEGASIS-TC. PEGASIS-TC exploits the spatial node redundancy in close knit sensor network due to random deployment. It selects a subset of nodes from the sensor field to ensure connectivity and form the chain based on Critical transmitting range $\mathrm{C}_{\mathrm{TR}}$. These left out nodes are switched on when their nearby nodes are drained out. This extends the lifetime of the sensor network. The simulation results illustrate that PEGASIS-TC outperforms PEGASIS.

The rest of the paper is organized as follows:

Section II explains radio energy dissipation model, section III gives the network model and assumptions used followed by section IV which describes PEGASIS-TC. Section V lists the performance metrics used for simulation. Section VI describes the results obtained. Finally section VII concludes the paper.

\section{RADIO ENERGY DISSIPATION MODEL}

In the radio energy model $[12,13]$, the transmitter dissipates energy to run the radio electronics and the power amplifier, and the receiver dissipates energy to run the radio electronics as shown in Figure 1.

Here both the free space $\left(d^{2}\right.$ power loss) and the multipath fading ( $d^{4}$ power loss) channel models are used, depending on the distance between the transmitter and receiver $[12,13]$. Power control can be used to invert this loss by appropriately setting the power amplifier-if the distance is less than a threshold $d_{o}$, the free space model is used; otherwise, the multipath model is used. 


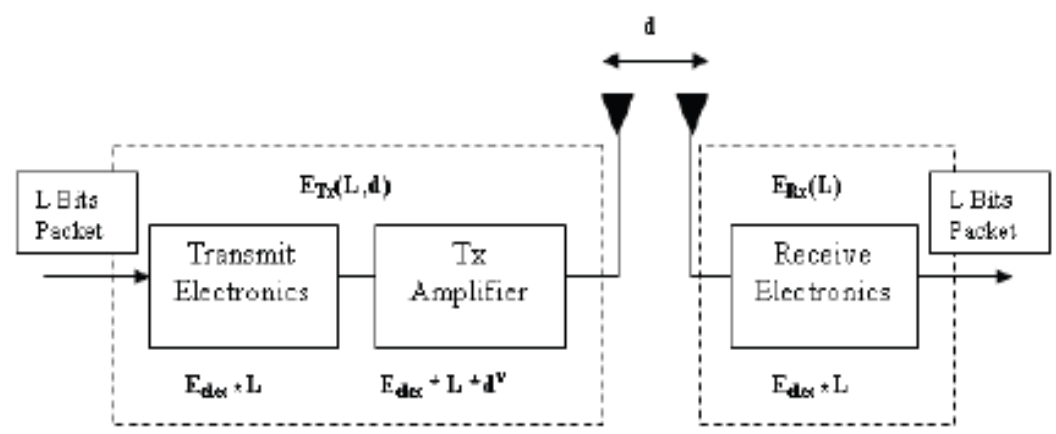

Figure 1. Radio Energy Dissipation Model

Thus, to transmit an L-bit message a distance, the radio expends

$E_{T x}(L, d)= \begin{cases}F \cdot E_{\text {elect }}+L \cdot E_{f s} \cdot d^{2} & \text { if } d<d_{o} \\ \text { L. } E_{\text {elect }}+L . E_{\text {amp }} \cdot d^{4} & \text { if } d>=d_{o}\end{cases}$

The electronics energy, $E_{\text {elec}}$, depends on factors such as the digital coding, modulation, filtering, and spreading of the signal, whereas the amplifier energy, $E_{\mathrm{fs}} \cdot \mathrm{d}^{2}$ or $\mathrm{E}_{\mathrm{amp}} \cdot \mathrm{d}^{4}$, depends on the distance to the receiver and the acceptable bit-error rate $[12,13]$. Value of threshold distance $d_{o}$ is given by

$$
\mathrm{d}_{\mathrm{o}}=\left(\mathrm{E}_{\mathrm{fs}} / \mathrm{E}_{\mathrm{mp}}\right)
$$

And to receive this message, the radio expends energy equivalent to

\section{NETWORK MODEL}

Our sensor network consists of 100 nodes in a $100 \times 100$ sensor field as shown in Figure 2.

For simplicity, we have taken following assumptions [14, 15]:

- All nodes are static.

- All nodes have power control capabilities, and each node can change the power level and communicate with BS directly.

- BS is located far away from the sensor field and at a fixed location.

- For a given signal to noise ratio, symmetric radio channel, making the energy required to transmit from one point to another and in reverse direction identical.

- Nodes always have data to send.

- Every sensor node generates a fixed size packet and forwards it to next node in the chain.

BS schedules transmission based on TDMA to avoid collision.

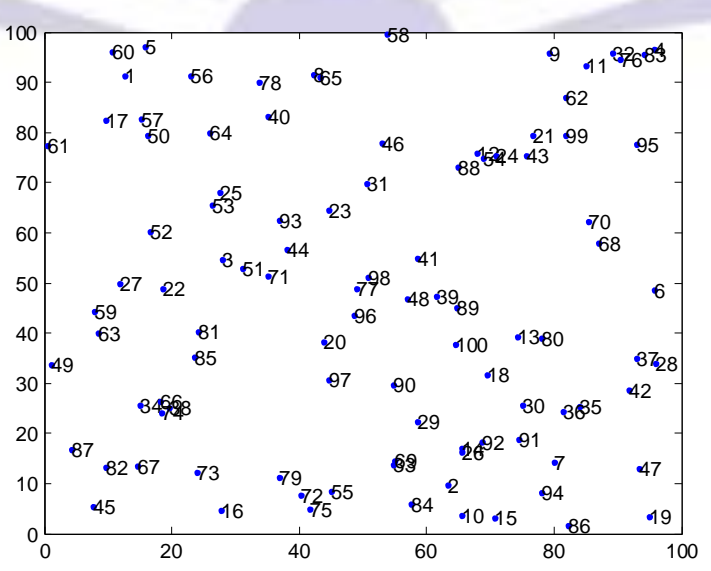

Figure 2. Random Wireless sensor network 


\section{PEGASIS-TC}

PEGASIS-TC is a improved chain based routing algorithm which operates in rounds. It consists of 3 stages: (1) Chain construction phase, (2) leader selection phase, (3) data transmission phase.

\section{A. Chain Construction Phase}

The algorithm uses the following steps to form a chain:

a) Initialize the network parameters. Determine the number of nodes, initial energy, BS location information et al. Then, the chain construction starts.

b) BS broadcasts the whole network a hello message to obtain basic network information such as ID of nodes alive, distance of each node to BS, and distance among the nodes.

c) Set the node farthest from BS as end node, it joins the chain first and is labeled node 1.

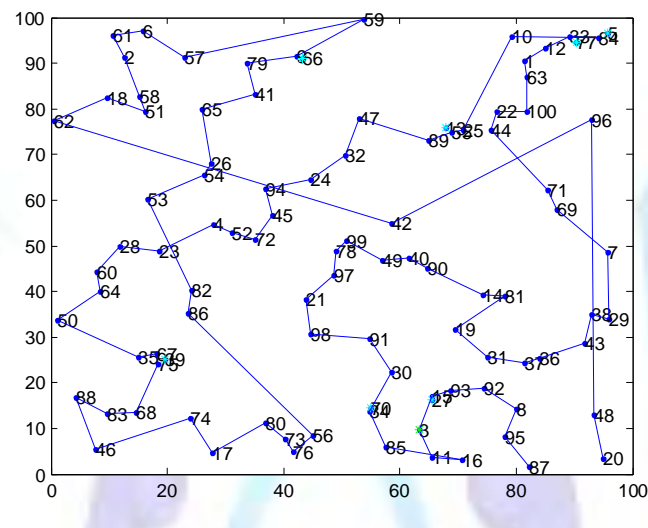

Figure 3. The chain formed in PEGASIS-TC

d) Calculate $\mathrm{C}_{\mathrm{TR}}$ as follows:

i. Sort all the distances between the nodes of the sensor field.

ii. Select $1 \%$ of minimum distances

iii. Compute their average as AVG

iv. Set $\mathrm{C}_{\mathrm{TR}}$ as $3 / 8^{*} \mathrm{AVG}$

e) Find the nodes which are at distance $=<\mathrm{C}_{T R}$ from end node.

f) Turn off the selected nodes.

g) Find minimum distant active node from end node.

h) Join it to chain \& make it next end node.

i) Continue steps e) to $h$ ) till all the nodes have been traversed.

The chain building scenario in PEGASIS-TC for a network of 100 nodes randomly arranged is shown in figure 3.

\section{B. Leader Selection Phase}

Leader selection in PEGASIS-TC is same as that of PEGASIS [11]. Node which transmits the data from the chain is called a leader. Leader will be in some random position $j$ on the chain. Nodes take turns transmitting to the BS, and will use node number $i$ mod $\mathrm{N}$ ( $\mathrm{N}$ represents the number of nodes) to transmit to the BS in round $i$. Therefore, the leader in each round of communication will be at a random position on the chain, for node deaths at random locations. This concept of random node deaths on the chain ensures robustness of the network towards failures.

\section{Data Transmission Phase}

Data transmission starts on successful construction of chain and leader node selection. Leader node initiates a token passing approach to start data transmission till the ends of the chain. Each node delivers its own sensed data to its neighbor node in the chain during their time slots assigned by TDMA mechanism. Then, the neighbor nodes fuse the received data with their own data \& forwards further towards the leader. One round will end until BS receives data from the leader. The sleeping nodes are activated and added to the chain as soon as nodes in $\mathrm{C}_{\mathrm{TR}}$ are drained out. In addition, it is assumed that chain is rebuilt when a node of the chain dies during simulation of experiment. 


\section{PERFORMANCE CRITERIA}

The number of Nodes Alive, number of Packets received at BS, Energy consumed per round \& Total Residual Energy of the sensor network are the performance parameters that have been used to study and evaluate the performance of the proposed protocol.

- Number of alive nodes: This instantaneous measure reflects the total number of nodes and that of each type that has not yet expended all of their energy.

- Data Packets received at base station: It is total number of data packets or messages that are received by the base station. This is also a measure of amount of information sent to BS from the sensor field. This measure varies linearly for all protocols.

- Energy consumed: It measures the instantaneous amount of energy being consumed in the network per round. This is simply the energy difference from the beginning till the end of a round.

- Network residual energy: It measures the total remaining energy of the network. It is calculated at each transmission round of the protocol.

The metrics used allow us to conclude about the stability of the network which is the time interval from the start of network operation until the death of the last sensor node. The lifetime of the network defined as the number of rounds until the last node die is simply the operational period of the network that is the period for which the network continues to provide information to the BS.

\section{RESULTS \& DISCUSSION}

Table 1: System parameters value

\begin{tabular}{|c|c|}
\hline Parameter & Value \\
\hline Network Size & $100 \times 100$ meter $^{2}$ \\
\hline Sink & $(50,300)$ \\
\hline Number of Nodes & 100 \\
\hline Initial Energy of Node & $0.50 \mathrm{~J}$ \\
\hline $\mathrm{E}_{\text {elect }}$ & $50 \mathrm{~nJ} / \mathrm{bit}$ \\
\hline $\mathrm{E}_{\mathrm{fs}}$ & $10 \mathrm{pJ} / \mathrm{bit}^{\mathrm{m}} \mathrm{m}^{2}$ \\
\hline $\mathrm{E}_{\mathrm{mp}}$ & $0.0013 \mathrm{pJ} / \mathrm{bit} / \mathrm{m}^{4}$ \\
\hline $\mathrm{E}_{\text {fusion }}$ & $5 \mathrm{~nJ} / \mathrm{bit} / \mathrm{message}$ \\
\hline Data Packet & $2000 \mathrm{bits}$ \\
\hline
\end{tabular}

This paper uses MATLAB as simulator to evaluate the performance of PEGASIS-TC. The system parameters used in the simulation are shown in the table $1[3,11,12,13]$.

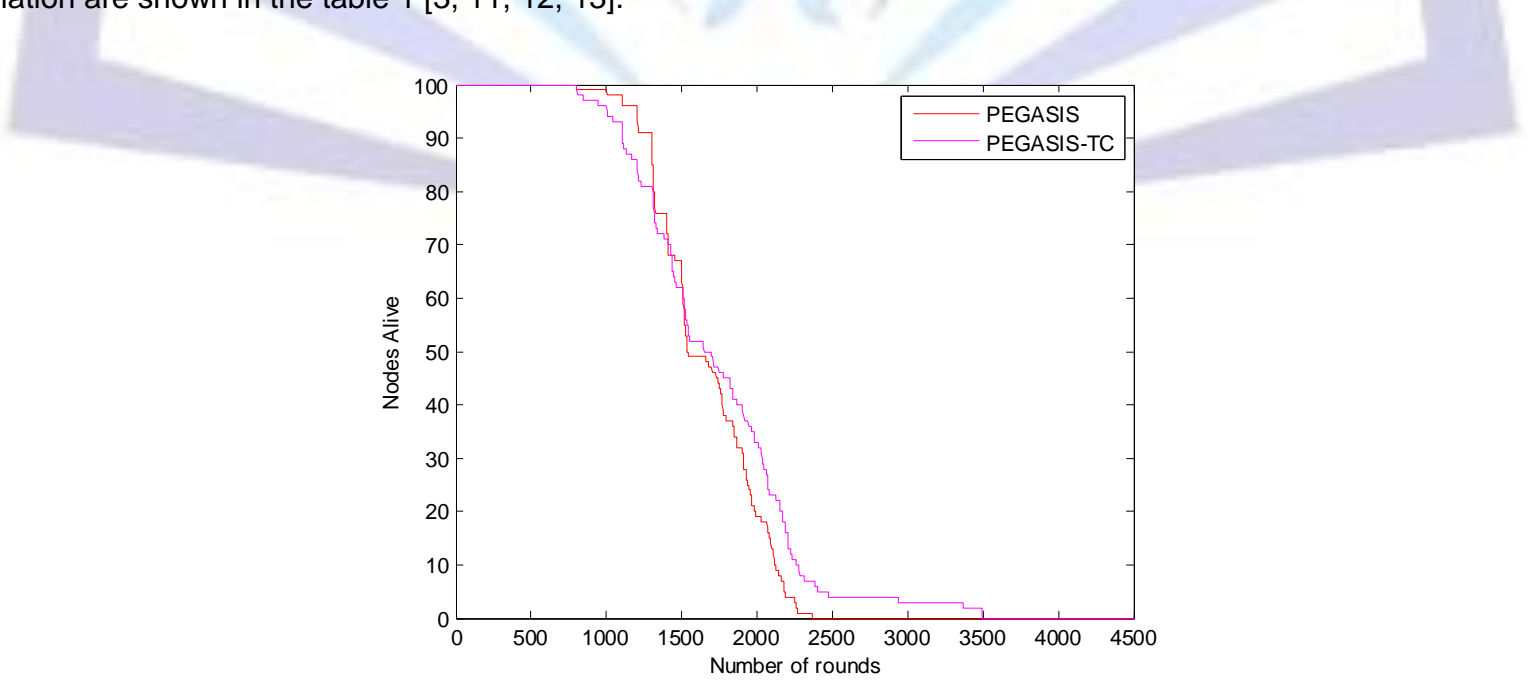

Figure 4. Number of nodes alive

Figure 4 depicts the number of alive nodes in the network. It is observed that PEGASIS-TC has a longer lifetime as its left out nodes are activated when a significant amount of nodes are drained out. While in PEGASIS these nodes' energy could 
have been drained to provide the information which is already available. This extends the sensor network lifetime. Moreover, PEGASIS-TC provides stability in lifetime as compared to PEGASIS. PEGASIS shows abrupt death of nodes due to almost same energy dissipation in all nodes, as they consume same amount of energy in each round. While PEGASIS-TC conserves the useful energy resource and uses it later.

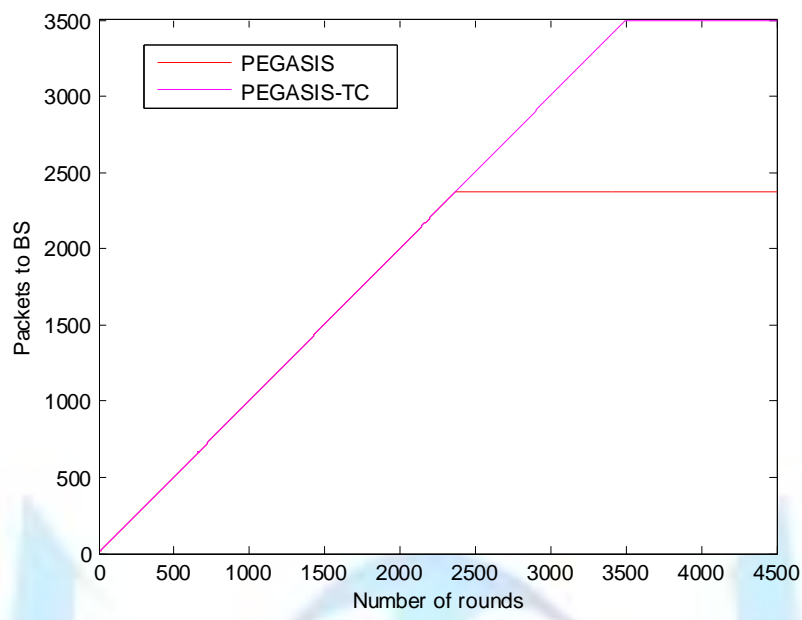

Figure 5. Number of Packets to BS

Figure 5 indicates a clear gain of $49 \%$ in number of Packets sent to BS for PEGASIS-TC as compared to PEGASIS [11]. It implies that amount of information to the base station is increased by a factor of 0.5 and may increase further based upon the deployment of sensor field. This is because the left out nodes which were switched off in earlier stages kept on providing the information, which could have been wasted in providing redundant information.

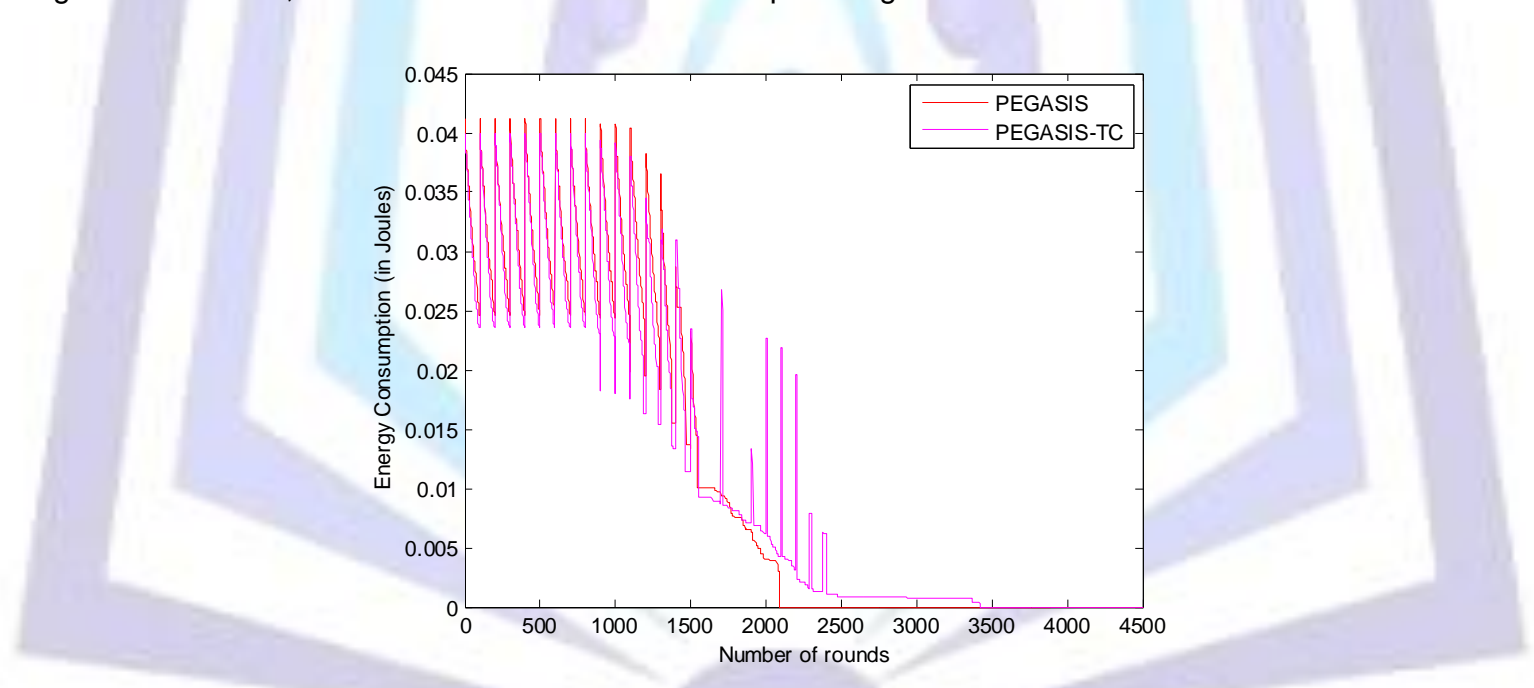

Figure 6. Energy consumed per round 


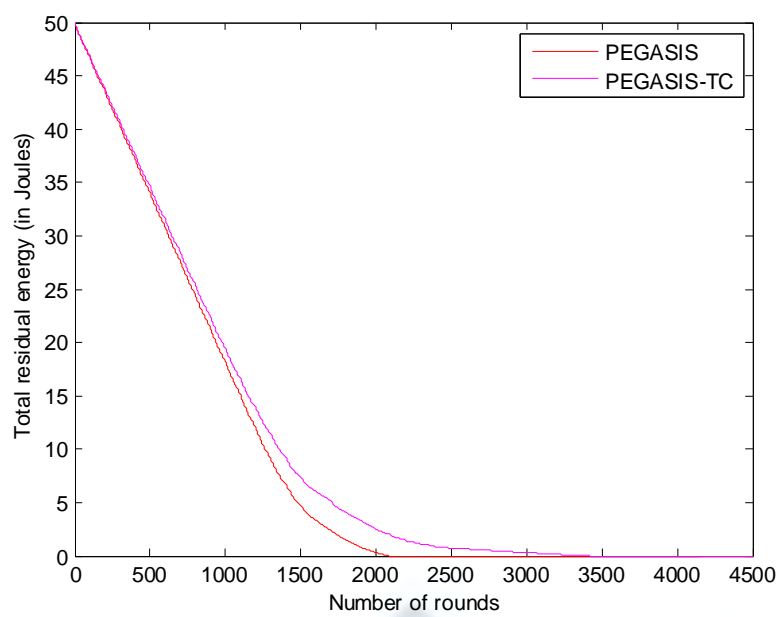

Figure 7. Total residual energy

Figure 6. shows energy consumption of PEGASIS-TC. It is concluded that PEGASIS-TC consumes less energy compared to PEGASIS because it has to chain lesser number of nodes to provide the information of whole sensor network. Lesser number of nodes to chain clearly indicates that less amount of energy to be spend to report the sensor field's information to the BS. This is the reason why PEGASIS-TC continues to a longer period of time.

The total residual energy per round in PEGASIS-TC is depicted in Figure 7. PEGASIS-TC miserly consumes energy instead of wasting it lavishly; therefore it continues to use its energy stock for a longer period of time.

\section{CONCLUSION}

This paper proposes energy proficient PEGASIS [11] based protocol PEGASIS-TC, which uses the concept of Topology control to manage the constrained energy resource. Node redundancy in Wireless Sensor Network leads to wastage of energy by providing the already available information. The novel algorithm turns off the node which lies in $\mathrm{C}_{T R}$ till there is a node to provide information. The energy thus saved is used later to prolong network lifetime. The simulation results prove that PEGASIS-TC outperforms PEGASIS [11] by achieving higher energy efficiency extending network lifetime.

\section{REFERENCES}

[1] Paolo Santi, "Topology Control in Wireless AdHoc and Sensor Networks", ACM Computing Surveys, Vol. 37, No. 2, June 2005, pp. 164-194.

[2] Giuseppe Anastasi, Marco Conti, Mario Di Francesco, Andrea Passarella, "Energy Conservation in Wireless Sensor Networks: A survey", Elsevier v.7 n.3, p. 537-568, May 2009 [doi: 10.1016/j.adhoc.2008.06.003]

[3] I.F. Akyildiz et.al.," Wireless sensor networks: a survey."Computer Networks, 2002, 38 (4) pp:393-422.

[4] Yunxia Chen and Qing Zhao, "On the Lifetime of Wireless Sensor Networks", Communications Letters, IEEE, Volume 9, Issue 11, pp:976-978, Digital Object Identifier 10.1109/ LCOMM.2005.11010., Nov. 2005.

[5] S. Lindsey, C. S. Raghavendra and K.Sivalingam, "Data Gathering in Sensor Networks using energy delay metric", In Proceedings of the $15^{\text {th }}$ International Parallel and Distributed Processing Symposium, pp 188-200,2001.

[6] V. Raghunathan, C. Schurghers, S. Park, M. Srivastava, "Energy-aware wireless microsensor networks", IEEE Signal Processing Magazine (2002) 40-50.

[7] G. Pottie, W. Kaiser, "Wireless integrated network sensors", Communication of ACM 43 (5) (2000) 51-58.

[8] D. Ganesan, A. Cerpa, W. Ye, Y. Yu, J. Zhao, D.Estrin, "Networking issues in Wireless sensor networks", Journal of Parallel and Distributed Computing 64 (2004) 799-814.

[9] A. Mainwaring, J. Polastre, R. Szewczyk, D. Culler, J. Anderson, "Wireless Sensor Network for habitat monitoring", in: Proc. ACM workshop on Wireless sensor networks and applications, Atlanta, USA, September 2002, pp. 88-97.

[10] A. Warrier, S. Park J. Mina, I. Rheea, "How much energy saving does topology control offer for wireless sensor network ?- a practical study, Elsevier/ ACM Computer Communication 30 (14-15) (2007) 2867- 2879.

[11] S. Lindsey, C.S. Raghavendra," PEGASIS: Power-efficient gathering in sensor information systems", IEEE Aerospace and Electronic Systems Society, Proc of the IEEE Aerospace Conf, Montana, 2002:1125-1130.

[12] W. Heinzelman, A. Chandrakasan, and H. Balakrishnan, "Energy-efficient communication protocols for wireless microsensor networks," Proceedings of the 33rd International Conference on System Sciences (HICS '00), January 2000. 
[13] W. Heinzelman, A. Chandrakasan, and H. Balakrishnan, "An application-specific protocol architecture for wireless microsensor networks", IEEE Transactions on Wireless Communications, Vol. 1, No. 4, pp. 660-670, October 2002.

[14] Jamal N. Al-Karaki, Ahmed E. Kamal, “ Routing Techniques in Wireless Sensor Networks: A Survey”, IEEE Wireless Communications, Volume: 11, Issue: 6,26-28, December 2004.

[15] Feng Sen, Qi Bing, Tang Liangrui, "An Improved Energy Efficient PEGASIS Based Protocol in Wireless Sensor Networks", IEEE 2011 Eighth International Conference on Fuzzy Systems and Knowledge Discovery (FSKD).

\section{Author' biography with Photo}

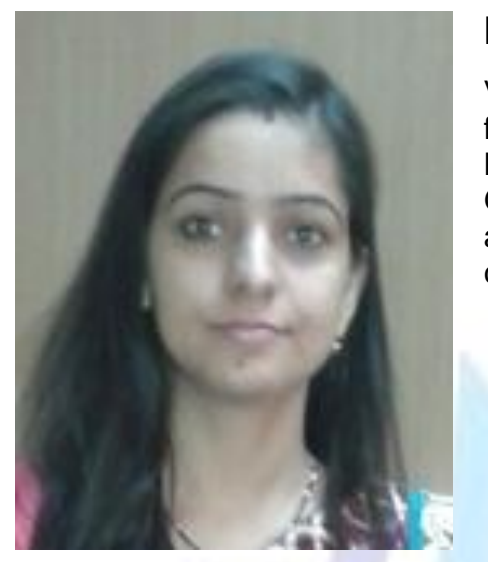

\section{Ms. Vibha Nehra}

Vibha Nehra obtained her M.Tech. in Department of Computer Science \& Engineering from Dr.B.R. Ambedkar National Institute of Technology, Jalandhar. She got her Bachelor" s degree in Computer Science \& Engg. from H.R. Institute of Technology, Ghaziabad, affiliated to Uttar Pradesh Technical University, Lucknow. Her research areas are Wireless Sensor Networks, Wireless \& Adhoc Network along with cryptography and Network Security.

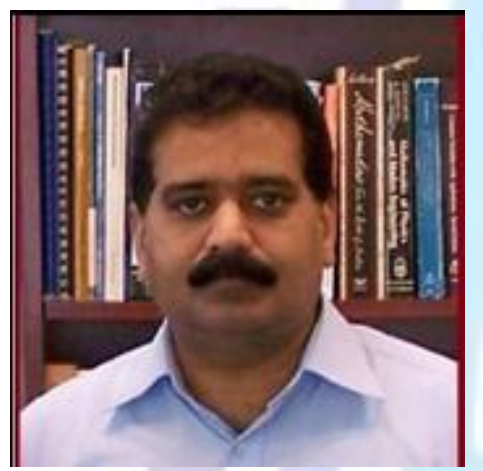

\section{Dr. Ajay K.Sharma}

Ajay K Sharma received his BE in Electronics and Electrical Communication Engineering from Punjab University Chandigarh, India in 1986, MS in Electronics and Control from Birla Institute of Technology (BITS), Pilani in the year 1994 and PhD in Electronics Communication and Computer Engineering in the year 1999. His PhD thesis was on "Studies on Broadband Optical Communication Systems and Networks". From 1986 to 1995 he worked with TTTI, DTE Chandigarh, Indian Railways New Delhi, SLIET Longowal and National Institute of Technology (Erstwhile Regional Engineering College), Hamirpur HP at various academic and administrative positions. He has joined National Institute of Technology (Erstwhile Regional Engineering College) Jalandhar as Assistant Professor in the Department of Electronics and Communication Engineering in the year 1996. From November 2001, he has worked as Professor in the ECE department and thereafter he has worked as Professor in Computer Science \& Engineering from 2007 to 2013 in the same institute. His major areas of interest are broadband optical wireless communication systems and networks, dispersion compensation, fiber nonlinearities, optical soliton transmission, WDM systems and networks, Radio-over-Fiber (RoF) and wireless sensor networks and computer communication. He has published 262 research papers in the International/National Journals/Conferences and 12 books. He has supervised 17 Ph.D. and 42 M.Tech theses. He has completed two R\&D projects funded by Government of India and one project is ongoing. He was associated to implement the World Bank project of 209 Million for TEQIP-I programme of the institute. He is technical reviewer of reputed international journals like: Optical Engineering, Optics letters, Optics Communication, Digital Signal Processing. He has been appointed as member of technical Committee on Telecom under IASTD Canada for the term 2004-2007 and he is Life member of Indian Society for Technical Education (I.S.T.E.), New Delhi. 